\title{
Experimental investigation of airflow above waves in a horizontal pipe
}

\author{
P. Vollestad ${ }^{1, *}$, A.A. Ayati ${ }^{1}$, L. Angheluta ${ }^{2}$, J.H. LaCasce ${ }^{3}$, A. Jensen ${ }^{1}$ \\ ${ }^{1}$ Department of Mathematics, University of Oslo, Norway \\ ${ }^{2}$ Department of Physics, University of Oslo, Norway \\ ${ }^{3}$ Department of Geosciences, University of Oslo, Norway \\ *Corresponding author.Tel.: +4748147276. E-mail: pettervo@math.uio.no.
}

\begin{abstract}
We investigate the effect of waves on the airflow in horizontal two-phase pipe flow. Velocity fields in the gaseous phase were acquired by particle image velocimetry (PIV), while interfacial elevation was measured with conductance wave probes. The velocity fields were sampled on a wave-following coordinate system which allows for a decomposition of the velocity field into a mean, wave-coherent and fluctuating component by means of a three-component Reynolds decomposition. Additionally, coherent vortical structures were identified by the swirling strength criterion, and their distribution along the waves is investigated.

Results suggest that the interaction between turbulent airflow and propagating waves in a pipe has a number of features reminiscent of wind-wave interaction in open systems. Above waves generated by sufficiently high gas flow rates, there is a distinct region of sheltered airflow, and a lifting of the critical layer on the leeward side of the crest. Streamlines of the phase-averaged flow field show a cat's eye structure located close to the crest in this region. Above waves of moderate steepness, we observe a shear layer that remains adjacent to the wave surface. Above steeper waves and higher gas flow rate, this layer detaches from the surface just downstream of the crest. Shear layer separation above waves is traditionally linked to the onset of wave breaking, and it is interesting to note that the case where we observe a separated shear layer in the phase-averaged vorticity field is in a regime of amplitude saturation.

The swirling strength criterion reveals that vortical structures are shed from the interface and populate the detached shear layer above the trough. Below the detached shear layer, there is a region populated by counter-rotating vortices. The critical height coincides with the border between these two regions.
\end{abstract}

Keywords: Two-phase pipe flow, Stratified flow, PIV, Vortical structures

\section{Introduction}

The interaction between a turbulent gas flow and propagating waves on a liquid layer plays an important role in the exchange of mass, momentum and heat across the interface. It is therefore an important problem to a wide range of research areas ranging from air-sea interactions to gas-liquid flow in pipes. The latter is relevant to engineering applications in petroleum, nuclear and process industries.

On the ocean surface, the rate at which momentum is exchanged between wind and waves depends on the wind to wave speed ratio (wave age). In situations where the waves are young, momentum is transferred from wind to waves by the action of form drag and viscous stresses, leading to wave growth and onset of surface currents (Grare et al., 2013). Proper understanding and modelling of these mechanisms is important to weather- and climate models as they rely on the parametrization of scalar and momentum fluxes across the air-sea interface (Janssen, 2004). A large number of experimental (e.g. Buckley
\& Veron (2016); Grare et al. (2013); Banner \& Peirson (1998); Reul et al. (2008)) and numerical studies (including LES (Large Eddy Simulations) and DNS (Direct Numerical Simulations, e.g. Sullivan et al. (2018); Hara \& Sullivan (2015); Yang \& Shen (2010)) has significantly improved our understanding of wind-wave interaction over the past decades. DNS has also been applied to study the initial development and growth of the wavefield under the action of wind (Lin et al., 2008; Zonta et al., 2015, 2016), where the wavenumber spectra of the initial capillary waves forming are found to follow the results from wave-turbulence theory (Deike et al., 2014; Pan \& Yue, 2014).

Turbulent gas-liquid flow in long pipes or channels includes many different flow regimes, from stratified to annular flow. The stratified regime occurs when gas and liquid velocities are relatively low; below the threshold for intermittent flow. If the gas velocity is sufficiently high, waves appear on the interface as a result of similar interfacial mechanisms as at the air-sea interface. The 
presence of waves leads to a complex two-way interaction between themselves and the turbulent gas flow. Depending on the gas-liquid velocity combination, different wavy flow patterns (sub-regimes) can be observed. Numerous investigators have studied and categorized the different sub-regimes, see for instance Tzotzi \& Andritsos (2013) and Fernandino \& Ytrehus (2006).

The main engineering aspect of the stratified regime consists of predicting flow parameters such as the pressure drop and liquid hold-up, as well as finding criteria for transition to slug flow. Engineers rely upon multiphase flow simulators (e.g. OLGA, see Bendiksen \& Espedal (1992) and LedaFlow) to simulate the flow inside pipelines over large distances. Naturally, the inherent complexity of the flow calls for significant simplifications of the problem in exchange for computational efficiency. The most common modelling approach is the socalled one-dimensional two-fluid model, that is, the velocity field in each phase is considered one-dimensional. Conservation equations are solved separately for each phase and connected at the interface through relevant kinematic and dynamic boundary conditions. This approach depends on closure relations for the interfacial friction. This particular step is a source of significant discrepancies between model predictions and experimental or field measurements. As a consequence, a large number of proposed friction factor correlations can be found in the literature, e.g. Andritsos \& Hanratty (1987) and Biberg (2007).

There is also a significant disparity in proposed mechanisms explaining interfacial wave growth and transition to slug flow. These include; i) linear stability of a stratified two-layer flow, e.g. one-dimensional viscous KelvinHelmholtz instability (Barnea \& Taitel, 1993) or twodimensional Orr-Sommerfeld analysis (Barmak et al., 2016; Kaffel \& Riaz, 2015), ii) local instability on top of a growing long wave (Kordyban \& Ranov, 1970; Woods et al., 2000) or, iii) non-linear wave interaction (Sanchis et al., 2011; Campbell \& Liu, 2016).

The lack of consensus on the subject calls for additional phenomenological studies exploring mechanisms that do not feature in present models. One of such mechanisms is flow separation above steep waves (Gent \& Taylor, 1977; Banner \& Melville, 1976). It is well known that airflow separation may have an important impact on interfacial momentum fluxes in turbulent gas-liquid flows (Makin \& Kudryavtsev, 2002; Banner \& Peirson, 1998).

Recent experimental studies using Particle Image Velocimetry (PIV) have led to a better understanding of the structure of the two-dimensional flow field in air-water flow in pipes (Ayati et al., 2014; Birvalski et al., 2014), and in large wind-wave channels (Reul et al., 2008; Veron et al., 2007; Buckley \& Veron, 2016). In previous studies, Ayati et al. (2014, 2015, 2016) combined PIV, conductance probing and hot-wire anemometry in order to acquire two-dimensional (2D) spatially resolved simultaneous measurements of both the gaseous and liquid flow fields, interfacial elevation statistics, and velocity fluctuations in the gaseous phase. The main outcome from the PIV measurements were presented in the form of spatiotemporally averaged velocity profiles. Such base flow profiles provide interesting flow information and can be used to conduct realistic stability analysis studies. However, they do not provide insight on the phase-dependency of the flow structure. This can only be achieved by conducting phase-locked measurements (Ayati et al., 2017) or by performing conditional-averaging techniques on statistically independent velocity fields. The latter is the scope of this paper.

Thus, in this study, we extend previous works by Ayati et al. by performing conditional phase-averaging on the gas-phase flow field, similar to the technique employed by Birvalski et al. (2014) and Siddiqui \& Loewen (2010) on the liquid phase. This enables us to decouple the wave-coherent fluctuations from residual fluctuations (turbulence and noise), and to study the phasedependency of the velocity field and the distribution of vortical structures along an average wave profile. Note that some alternative results from this analysis has been presented in a conference proceeding (Ayati et al., 2018), highlighting main results from different studies on the topic of two-phase stratified pipe flow.

In order to keep the paper concise, we conduct a detailed comparison of two different flow cases in which the liquid superficial velocity is kept constant at $U_{s l}=0.1$ $\mathrm{m} / \mathrm{s}$, whilst the gas velocity increases from $U_{s g}=1.5$ in case A, to $2.1 \mathrm{~m} / \mathrm{s}$ in case B. These cases were selected as they feature in two different sub-regimes, as shown by Ayati \& Carneiro (2018). Case A belongs to a region of flow conditions in which waves grow as a consequence of increasing gas flow rates. In this regime, interfacial elevation statistics are well described by the Gaussian wave model (Onorato et al., 2013), indicating that these waves behave quasi linearly. Meanwhile, case $\mathrm{B}$ belongs to a regime in which wave amplitudes remain more or less constant within a wide range of gas velocities above $U_{s g}=1.75 \mathrm{~m} / \mathrm{s}$. In this regime, the waves are steeper and their statistical distributions deviate strongly from Gaussian statistics, implying the presence of nonlinear processes (Ayati \& Carneiro, 2018). Similar results have been reported by Toffoli et al. (2017) for a circular, fetch-unlimited wind-wave tank. They also observed that waves in a growth regime were well represented by Gaussian statistics, while strong deviations from Gaussian statistics were observed as the wave field reached a stationary state.

Saturation of wave growth in confined gas-liquid flows has been addressed by several investigators, see for instance Jurman et al. (1992) and Campbell et al. (2016). They both attributed this phenomenon to non-linear energy transfer from linearly unstable to stable interfacial modes through sub-harmonic and triadic resonances. Although these are sophisticated approaches, they do not address the role of modified momentum flux due to changes 
in the gas flow structure. Thus, a more specific motivation for this study is to investigate whether the proposed phase-averaging technique can reveal new dynamics in the gas flow field that may further explain this sub-regime transition.

This paper is organized as follows; the experimental setup and data processing methodology are presented in section 2. Results and discussions are presented in section 3, which includes instantaneous and phase-averaged flow fields and an investigation of the phase-distribution of vortical structures. Finally, concluding remarks are outlined in section 4 .

\section{Experimental setup and methodology}

\subsection{Experimental setup}

The data under investigation were acquired during an experimental campaign conducted at the Hydrodynamics Laboratory, University of Oslo. The experimental techniques (PIV, conductance probing and hot-wire anemometry) were combined to study air-water flow in pipes (Ayati et al., 2014, 2015, 2016). Here, we will primarily focus on the data stemming from PIV in the air phase, and use some of the results from the wave gauges.

A $31 \mathrm{~m}$ long horizontal acrylic pipe with internal diameter D $=100 \mathrm{~mm}$ was used in the experiments, the test fluids were air and water at atmospheric pressure. Fluorescent dye (Rhodamine B) was added to the water in order to increase the intensity jump across the interface (enabling efficient interface detection from the PIV images) and minimize local light reflections. Both fluids were introduced at the pipe inlet using frequencyregulated pump and fan, for the water and gas, respectively. A plate separating the two phases at the inlet caused the liquid phase to be introduced without waves, these developed along the pipe due to the airflow above the liquid interface. For each experimental case considered the gas and liquid flow rates were kept constant for some time before the measurements started, ensuring that the statistical properties of the flow were constant at the measurement section during the experiments. The water and air mass flow rates were measured with an Endress Hauser Promass and an Emerson MicroMotion Coriolis flow meter, with $0.2 \%$ and $0.05 \%$ of maximum measured values in accuracy, respectively. A schematic view of the experimental setup is shown in figure 1 .

A wave gauge was placed approximately $270 \mathrm{D}$ downstream from the pipe inlet. The gauge consisted of two double-wire probes made of platina wires of $0.3 \mathrm{~mm}$ diameter and separated by $4 \mathrm{~mm}$. Both probes were placed in the center of the pipe with a distance $d=60 \mathrm{~mm}$ in the streamwise direction, enabling calculation of the wave speed through cross-correlation methods. Interface elevation was measured with a relatively high temporal resolution of $500 \mathrm{~Hz}$. For more details about the interface elevation measurements, the reader is referred to Ayati et al. (2015).
Simultaneous two-phase PIV (S2P-PIV) was conducted along the pipe centerline in a PIV section located approximately 260D downstream from the pipe inlet. A doublepulsed Nd:YAG laser of $135 \mathrm{~mJ}$ illuminated a vertical plane along the pipe centerline from above. The images were recorded with two 14-bit PCO.4000 cameras (one for each phase). The gas-phase camera was located 15 $\mathrm{cm}$ above the pipe centerline, tilted $15^{\circ}$ downwards, with a horizontal field of view (FOV) of $90 \mathrm{~mm}$. The gas phase was seeded with small water droplets, injected at the pipe inlet using a high-pressure atomizing nozzle. The nozzle produces small particles (according to the manufacturers specifications, $72 \%$ of the droplets are below $6 \mu \mathrm{m}$ ), which are found to behave as passive tracers for the flow rate combinations under investigation (Ayati et al., 2014). Double images with a time delay of 250-300 $\mu$ s (depending on the flow rate combinations) were acquired at a rate of $0.3 \mathrm{~Hz}$. For each experimental case, 1000 image pairs were obtained from the experiments. The S2P-PIV has been described in more detail by Ayati et al. (2014). In the present work only the images of the air-phase are analyzed.

For the present study the air phase PIV images have been re-analyzed using an in-house PIV routine based on cross-correlation (Kolaas, 2016). A cascade of crosscorrelation passes with increasingly fine subwindow sizes was applied. The final subwindow size was 40x20 pixels, with $75 \%$ overlap. This resulted in a spatial resolution of approximately $0.25 \times 0.25 \mathrm{~mm}^{2}$. Subpixel interpolation is performed with a $3 \times 3$ gaussian subpixel estimator, and outliers are detected and replaced by means of a $3 \times 3$ local median filter. The fraction of spurious vectors were maximum near the interface, typically in the range of 15 $\%$. The results from the present cross-correlation procedure has been compared with the results obtained by Ayati et al. (2016). The results for the mean flow structures are similar, and no qualitative differences are found on the phase-averaged velocities presented in this paper comparing the two sets of results. However, due to higher spatial resolution, it is possible to extract more details of the small scale vortical structures from the updated velocity profiles, presented in section 3.3.

\subsection{Conditional phase-averaging procedure}

In this section the conditional phase-averaging routine is described. The first step in the analysis is to detect the interface between the gas and liquid phase. The methodology used by Ayati et al. (2015), in which the interface is detected from the differences in light intensity across the water interface of the PIV images was used also here, and a manual control of all the detected interfaces was performed.

The field of view, which was $90 \mathrm{~mm}$ in width, was not wide enough to fully resolve the dominant wavelengths present. These were in the range of 1.8 to 2.5 times the FOV width. For this reason the interface was divided into wave quadrants, and the averaging was performed for a 


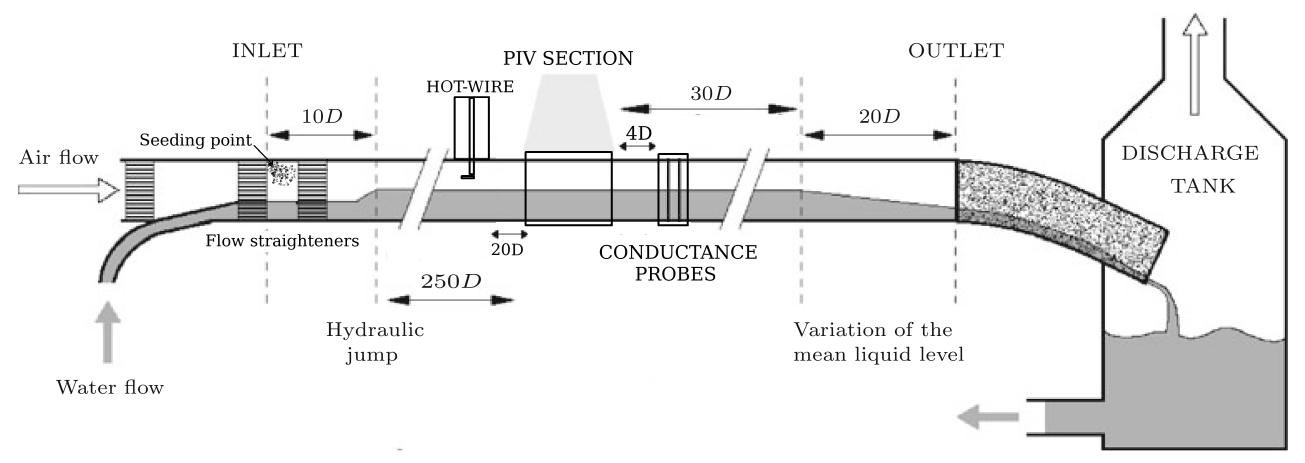

Figure 1: Schematic view of the experimental setup in use. Figure from Ayati et al. (2016).

sequence of velocity fields related to the same quadrant. A similar approach was employed by Siddiqui \& Loewen (2010) and Birvalski et al. (2014), averaging the liquid phase beneath a wavy interface.

Wave quadrants were identified by means of a zerocrossing procedure. Between two consecutive zero-crossings, global maxima/minima were identified as crests/troughs. If a local maxima/minima (which is not on the edge of the PIV image) is detected to the left (right) of the first (last) zero-crossing of a PIV image, this is also assessed to be a crest/trough of a wave. The region in between a zerocrossing and a crest/trough was then classified as a wave quadrant according to the definition in figure 2. A minimum wave amplitude of $1 \mathrm{~mm}$ was used as a threshold in order to limit the influence of measurement uncertainties close to the interface (due to strong light reflections near the interface, see Ayati et al. (2014)). This threshold also governs that the minimum wavelength considered is approximately $50 \mathrm{~mm}$, ensuring that all waves considered are gravity dominated waves (Birvalski et al., 2015).

The air domain was subjected to a coordinate transformation from Cartesian $(x, y)$ to wave-following coordinates $(\theta, \zeta)$. The crest, trough and zero-crossings were assigned phases of $0^{\circ}, 180^{\circ}$ and $+/-90^{\circ}$, respectively (see figure 2), whilst phases in between were linearly distributed. The vertical coordinate $\zeta=(y-\eta(x)) / \eta_{c}$ is the dimensionless height above the interface, normalized by the crest height. The resulting coordinate system is illustrated in figure 2. Velocity fields above the identified wave quadrants are sampled from $\zeta=0$ to 5 . For each quadrant observed, all variables of the flow field $(u$, $v$, vorticity etc.) were sampled at the equally distributed $(\theta, \zeta)$ coordinates, with 180 points in the $\theta$-direction and 100 points $\zeta$-direction.

The normalization of the vertical coordinate ensures that points in the trough-to-crest region are averaged with other points located at the same relative position in the trough-to-crest region. Outside of this region, where the wave amplitude is no longer a characteristic length scale of the flow, the normalization is less relevant. For this reason, the phase-averaging performed in the present work is mostly valuable in the crest-to-trough region. Alter- native wave following coordinate systems have been applied by, amongst others Buckley \& Veron (2016); Hara \& Sullivan (2015); Hsu et al. (1981), for flow above water waves in open channels. The advantage of these systems is that the vertical coordinate becomes horizontal , when far away from the interface, i.e., $\zeta \rightarrow y$ as $y$ becomes large. This removes artificial wave-coherent fluctuations far from the interface, caused by the varying vertical position of the wave following coordinate system in the undisturbed region of the flow. As in the present study we are primarily interested in the region very close to the interface, the proposed coordinate system is assessed to be suitable.

As the wave field consists of a spectrum of wave components with varying heights, lengths and steepnesses (see figure 3 ), a criterion is employed prior to averaging. The wave steepness is known to have a significant impact on the airflow above waves, affecting the critical layer thickness, streamline patterns and potential for airflow separation above the waves (Gent \& Taylor, 1977; Reul et al., 2008). For this reason, quadrant steepness was chosen as the averaging criterion in this study. The steepness of each observed quadrant is defined as $\epsilon=\eta_{c t} / \lambda_{q}$, where $\lambda_{q}$ is the quadrant length and $\eta_{c t}$ is crest/trough height relative to the mean water level. Typical scatter plots of quadrant lengths and crest/trough heights are shown in figure 3. Wave quadrants of equal steepness are found along straight lines from the origin. Thus, by averaging over realizations along different lines, we may evaluate the influence of steepness on the airflow. In order to achieve a valuable number of averaging realizations, a steepness range is defined as $\epsilon_{0} \pm \alpha \epsilon_{0}$, where $\alpha$ represents fractional variation in steepness. In the present study, $\alpha$ was adjusted such that a minimum of 40 quadrant observations were included in each averaging window. This was found to be a sufficient number for achieving a qualitative convergence of the velocity fields.

Typical interface profiles selected on the basis of the steepness criteria are shown in figure 4 . The variability in the interfaces detected is clearly visible. These variations have the effect of inducing an additional fluctuation into the fluctuating component of the decomposed veloc- 


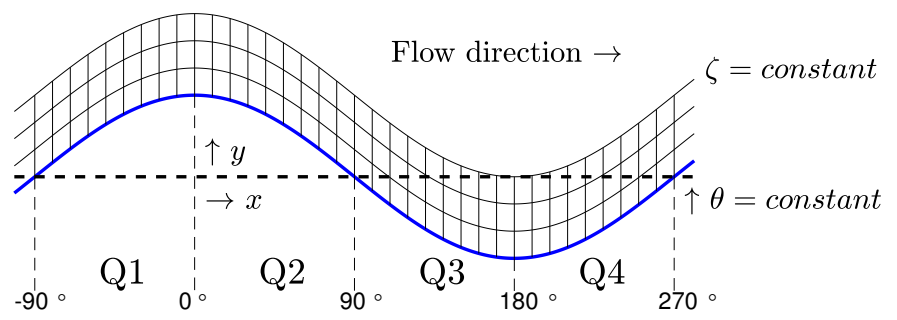

Figure 2: Definition of wave quadrants and illustration of wave-following coordinate system applied. Blue line: Water interface. Dotted horizontal line: Mean water level. Wave following coordinate system $(\theta, \zeta)$ illustrated above the water interface.
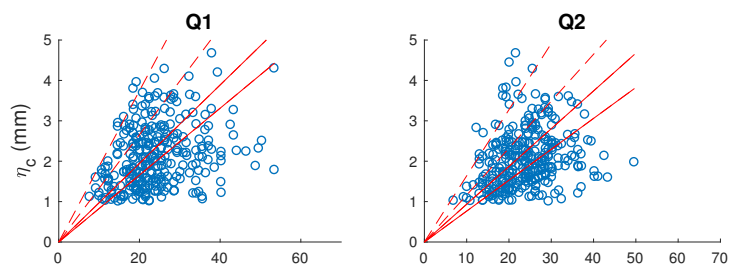

Q3
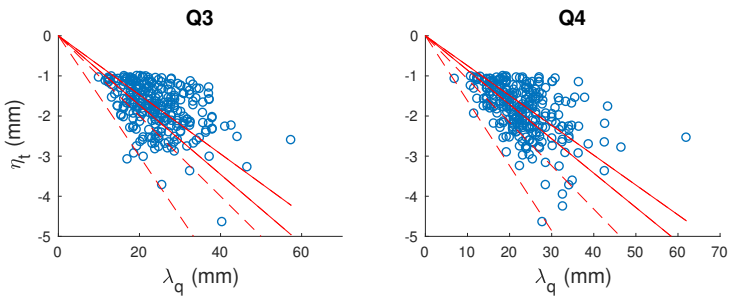

Figure 3: Typical scatter plot of quadrant lengths and crest/trough heights for one experimental case. Red lines indicate two ranges of steepness criteria; median steepness (solid lines) and high/maximum steepness (dashed lines).

ity field (see section 2.3).

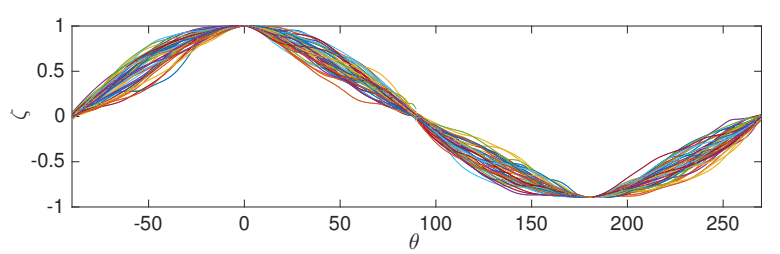

Figure 4: Quadrant interface profiles selected by means of the steepness criteria. Interfaces normalized according to wave-following coordinate system.

\subsection{Three-component Reynolds decomposition}

A three-component Reynolds decomposition is applied on the averaged velocity fields (Hsu et al., 1981; Birvalski et al., 2014; Buckley \& Veron, 2016). For a given variable $q(x, y, t)$ in the flow field, the variable is decomposed into a phase independent mean $\bar{q}(\zeta)$, a waveinduced field $\tilde{q}(\theta, \zeta)$ and a fluctuating component $q^{\prime}(\theta, \zeta, t)$ :

$$
q(x, y, t)=\bar{q}(\zeta)+\tilde{q}(\theta, \zeta)+q^{\prime}(\theta, \zeta, t)
$$

The decomposition is illustrated in figure 5 for the horizontal velocity component $u$ in a single Q2 observation. The phase-averaged variable $\langle q\rangle(\langle q\rangle=\bar{q}+\tilde{q})$ is obtained by averaging several instantaneous velocity fields selected by the steepness criterion. $\bar{q}$ is evaluated based on $\langle q\rangle$ for all four wave quadrants. The wave-coherent component is then given as $\tilde{q}=\langle q\rangle-\bar{q}$, while the fluctuating component is evaluated as $q^{\prime}=q-\langle q\rangle$. The fluctuating quantities $u^{\prime}$ and $v^{\prime}$ are often referred to as turbulent fluctuations (Birvalski et al., 2014; Buckley \& Veron, 2016). However, as the averaging is performed over a spectrum of waves the fluctuations should rather be interpreted as a combination of turbulence and fluctuations caused by variations of the air-water interface and the coordinate system applied.

Our focus will be on the phase-averaged $(\langle q\rangle)$ and wave induced $(\tilde{q})$ components of the velocity field above the waves. The influence of interface variations on the fluctuating velocity components, coupled with a limited dataset, means that the Reynolds stresses (e.g. $\overline{u^{\prime} v^{\prime}}$ ) are not considered to provide a reliable measure for the turbulence. In order to get an impression of the variations of the small scale turbulent fluctuations along the wave, we extract vortical structures and investigate their variations over the waves (see section 3.3).

\subsection{Experimental cases}

Two experimental cases performed by Ayati et al. (2016) are revisited. The main characteristics of the two experimental cases are presented in table 1 . Here $U_{s f}, U_{b f}$, $R e_{D f}$, with subscript $f=g, l$ indicating gas or liquid, are the superficial velocities, bulk velocities and Reynolds numbers which are based on the bulk velocity and hydraulic diameter. The relationship between bulk and superficial velocity is $U_{b f}=U_{s f} A / A_{f}$, where $A_{f}$ is the cross-sectional area occupied by fluid $f$. Furthermore, $\eta_{r m s}, c, \lambda_{p}, \bar{H}_{w}$ and $c / u_{*}$, are the root-mean-squared interface elevation relative to the mean water level (interface evaluated from PIV images), characteristic wave celerity given by means of cross-correlation of conductance probe signal, dominant wave-length based on the peak interfacial spectral density and wave celerity, mean liquid height and wave age, respectively.

The wave age $c / u_{*}$ indicates that the waves are young, wind driven waves (Buckley \& Veron, 2016; Belcher \& Hunt, 1998). The friction velocity $u_{*}=\sqrt{\tau_{i} / \rho_{g}}$ was determined through the momentum balance equation, in which interfacial shear stress $\tau_{i}$ is balanced by the pressure drop and wall-friction. The pressure drop was mea- 

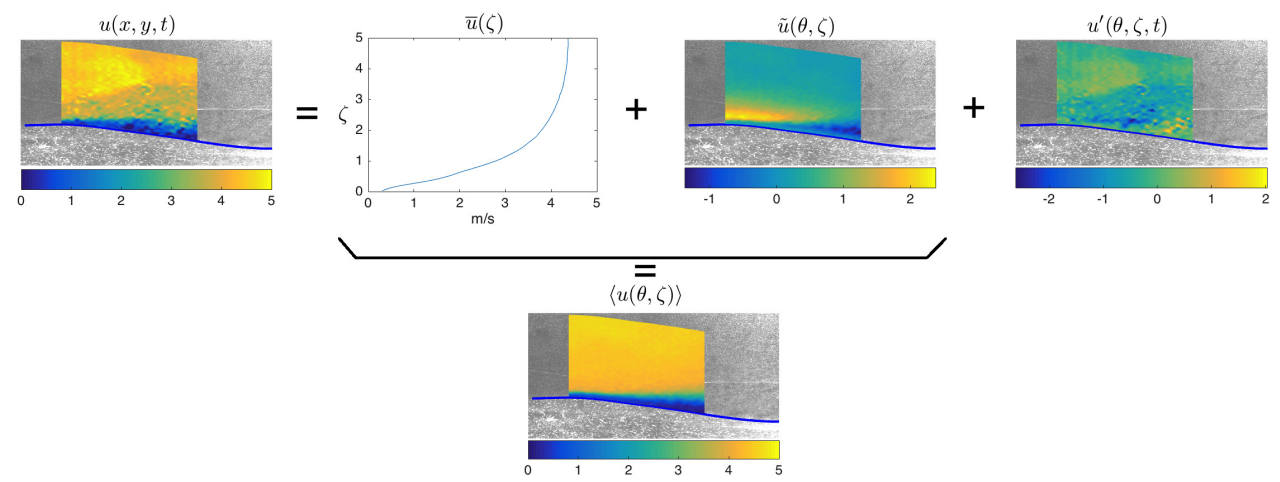

Figure 5: Illustration of the three-component decomposition of the horizontal velocity field applied in the study. Colorbars are in $\mathrm{m} / \mathrm{s}$. Velocity fields of identified quadrant overlaid original PIV image.

\begin{tabular}{|c|c|c|c|c|c|c|c|c|c|c|c|}
\hline Exp. case & $\begin{array}{c}U_{s l} \\
{[\mathrm{~m} / \mathrm{s}]}\end{array}$ & $\begin{array}{c}U_{s g} \\
{[\mathrm{~m} / \mathrm{s}]}\end{array}$ & $\begin{array}{c}U_{b l} \\
{[\mathrm{~m} / \mathrm{s}]}\end{array}$ & $\begin{array}{c}U_{b g} \\
{[\mathrm{~m} / \mathrm{s}]}\end{array}$ & $\begin{array}{c}R e_{D g} \\
{[-]}\end{array}$ & $\begin{array}{c}R e_{D l} \\
{[-]}\end{array}$ & $\begin{array}{c}\eta_{r m s} \\
{[\mathrm{~mm}]}\end{array}$ & $\begin{array}{c}c \\
{[\mathrm{~m} / \mathrm{s}]}\end{array}$ & $\begin{array}{c}\lambda_{p} \\
{[\mathrm{~m}]}\end{array}$ & $\begin{array}{c}\bar{H}_{w} \\
{[\mathrm{~mm}]}\end{array}$ & $\begin{array}{c}c / u_{*} \\
{[-]}\end{array}$ \\
\hline $\mathrm{A}$ & 0.1 & 1.5 & 0.26 & 2.44 & 11200 & 25200 & 1.25 & 0.71 & 0.16 & 42 & 2.7 \\
\hline $\mathrm{B}$ & 0.1 & 2.1 & 0.26 & 3.49 & 15800 & 24900 & 3.00 & 0.81 & 0.22 & 41 & 2.1 \\
\hline
\end{tabular}

Table 1: Experimental cases under investigation.

sured over a 12.3 meter section of the pipe, while the wall friction was estimated fitting a logarithmic profile to the mean velocity profile in the log-layer near the upper pipe wall $\left(30<y^{+}<80\right)$. Different methods for calculating the interfacial friction exists, providing slightly different results. Estimating the wall friction based on the Colebrook-White equation resulted in a $10 \%$ deviation from the present method. While the calculated $u_{*}$ is assessed to give a reasonable estimate for the wave age, and display clearly that we are in a regime of young, wind driven waves $\left(c / u_{*}<5\right.$ considered young waves), $u_{*}$ should be used with care, as crosswise and spanwise variations in the wavefield means that the interface friction will vary along the pipe section. As the interface friction is estimated based on a pressure drop evaluated over a 12.3 meter section of the pipe, this represents a characteristic interface friction for the system, rather than for the centerplane of the PIV section.

Detailed analysis of the evolution of the wave field with different $U_{s l} / U_{s g}$-combinations has previously been performed by Ayati et al. (2015) and Ayati \& Carneiro (2018). Here it has been demonstrated that case A is in a region of the flow map where $\eta_{r m s}$ increases with increasing gas flow rates, while case B is in a region of "amplitude saturation", where $\eta_{r m s}$ is independent of the gas flow rate. Example time-series of the interface elevation measurements are presented in figure $6 a-b$ ), while frequency spectra of the two experimental cases is presented in figure $6 \mathrm{c}$ ). Turbulence spectra in the air-phase (evaluated by hot-wire anemometry) has previously been presented by Ayati et al. (2016). These reveal an inertial range (-5/3 log-law), characteristic of a turbulent flow, and distinct low-frequency peaks related to the propagating waves.

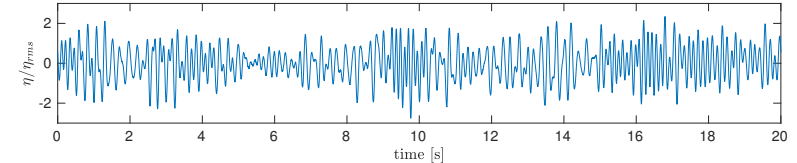

a) $U_{s g} 1.50 \mathrm{~m} / \mathrm{s}$.

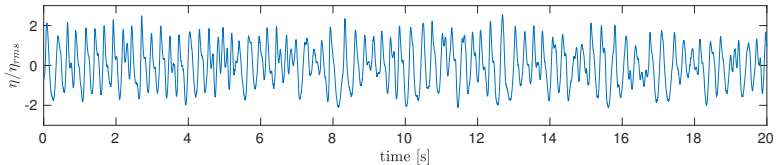

b) $U_{s g} 2.10 \mathrm{~m} / \mathrm{s}$

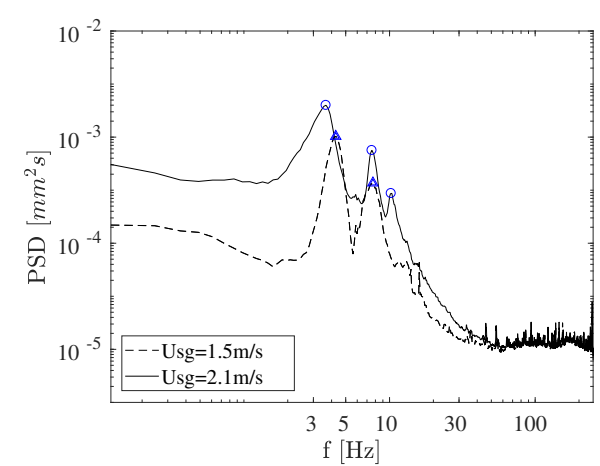

c) Frequency spectra

Figure 6: a-b) Example interfacial elevation measurements for the two experimental cases. Normalized by $\eta_{r m s}$. c) Interfacial power spectral density (PSD) for the two experimental cases.

From the two experimental cases, a total of three averaging cases (based on quadrant steepness) are analyzed. These are referred to as A1, A2 and B1. Details on the averaging cases are presented in table 2 and 3 . Note that the experimental case A has two averaging cases, where A1 represents wave quadrants with median steepness, and 
A2 represents waves with the maximum steepness observed. Case B only has one averaging case (B1), representing waves with the median steepness.

From table 3, it can be seen that the waves at $U_{s g}=$ $1.50 \mathrm{~m} / \mathrm{s}$ (case A1 and A2) are fairly symmetric around the mean, whereas at $U_{s g}=2.1 \mathrm{~m} / \mathrm{s}$ (case B1), they have tall and narrow crests and long and shallow troughs. This indicates that in case B1, the waves have some degree of non-linearity. This is in line with the characterization of the wave field by Ayati \& Carneiro (2018), where it is found that the $U_{s g}=1.50 \mathrm{~m} / \mathrm{s}$ case behaves according to Gaussian statistics, while the $U_{s g}=2.1 \mathrm{~m} / \mathrm{s}$ case deviates significantly from linear theory. Furthermore, the second quadrant of case B1 is considerably shorter and steeper than the other quadrants. While case A2 has a higher wave steepness $(a k)$ than case B1, the Q2 steepness is higher for case B1. This is considered to be particularly important to airflow separation and possible microscale breaking events.

\section{Results and discussion}

The results section is divided into three parts. First, instantaneous velocity fields for each averaging cases are presented in section 3.1. Secondly, phase-averaged velocity fields are shown in section 3.2, and lastly, the distribution of vortical structures along an average wave profile is investigated in section 3.3.

\subsection{Instantaneous flow fields and intermittent airflow sep- aration}

In figure 7 the horizontal velocity and the spanwise vorticity over waves with a Q2 steepness matching the intervals used for the three averaging cases are presented. Figure 7 a) and b) demonstrate the flow field dependency on the Q2 steepness. At low steepness, both the velocity and vorticity fields are relatively unaffected by the wave, except for a small region at $\mathrm{x}=[30-40] \mathrm{mm}$ where a small undulation induces extra vorticity. This particular observation is interesting as it shows the effect of surface roughness caused by small waves riding on top of long waves. In general, the shear layer is seen to remain adjacent to the water surface. At higher steepness but same air velocity (figure $7 \mathrm{~b}$ ), a region of sheltered flow is more visible behind the crest. The vorticity field shows sign of detachment at $x \approx 0 \mathrm{~mm}$. These features are more pronounced in figure $7 \mathrm{c}$ ), where both the air velocity and wave steepness are higher. Herein, the region of sheltered flow is considerable and negative axial airflow is observed on the leeward side of the crest. The negative vorticity layer is seen to separate from the crest and a region of positive vorticity is seen above the trough, between the water surface and the separated shear layer. The phase-averaged statistics of the vorticity field will be investigated in section 3.3.2.

Airflow separation has traditionally been linked to the onset of wave breaking (Gent \& Taylor, 1977; Banner \&
Melville, 1976; Reul et al., 2008). Recent experimental (Veron et al., 2007) and numerical (Sullivan et al., 2018) analysis does however indicate that while airflow separation is a sufficient criterion for separation, it is not a necessary criterion, as airflow separation has been reported over non-breaking waves.

Buckley \& Veron (2016) considered the airflow as separating "if the near-surface, high vorticity layer characteristic of an attached boundary layer is ejected away from the water surface and the surface vorticity is near zero or negative" 1 . Figure 7 c) demonstrates that (instantaneous) separation of the shear layer occurs for case B1. While no strong breaking (breaking with air entrainment) was observed during the experiments, we are currently not able to assess whether weak forms of wave breaking (microscale breaking) coincides with the airflow separation observed in figure $7 \mathrm{c}$ ). The steep wave-fronts observed for the $U_{s g} 2.10 \mathrm{~m} / \mathrm{s}$ case (ref. section 2.4) is a strong indicator of wave breaking, and visual observations of the waves at $U_{s g}=2.10 \mathrm{~m} / \mathrm{s}$ indicate that weak forms of wave breaking is intermittently occuring. However, as demonstrated by Siddiqui \& Loewen (2006), while microbreaking is well correlated with high wave steepness, the maximum wave slope is not a sufficient criterion for detecting breaking. Hence, at this stage we are not able to quantify the frequency of small-scale wave breaking in the system, or relate these events directly to the observed airflow separation.

\subsection{Phase-averaged velocities}

The phase-averaged horizontal $(\langle u\rangle)$, vertical $(\langle v\rangle)$, mean horizontal $(\bar{u})$ and the wave-coherent horizontal velocity $(\tilde{u})$ for averaging cases $\mathrm{A} 1$, $\mathrm{A} 2$ and $\mathrm{B} 1$ are presented in figure 8, plotted above the mean interface evaluated for each averaging case. The critical height $z_{i}$ (defined as the height where $\langle u\rangle=c$ ) is indicated by a reddotted line in the $\langle u\rangle$ fields. Note that the mean vertical velocity profile and vertical wave-coherent field are not shown to avoid overcrowding the figures. The former is nearly zero, and as $\tilde{v}=\langle v\rangle-\bar{v}$ it will be nearly equal to $\langle v\rangle$.

Figure 8 a) and b) show that with constant wind conditions, higher wave steepness affects both the phase-averaged vertical velocity $\langle v\rangle$ and the wave-coherent horizontal velocity $\tilde{u}$. Larger steepness induces stronger positive $\langle v\rangle$ upstream of the crest and stronger negative $\langle v\rangle$ above the the trough. Also $\tilde{u}$ is stronger above the crest and trough.

As expected from the instantaneous flow fields (section 3.1), a significant sheltered region is observed behind the crest of case B1 (figure $8 \mathrm{c}$ ). Furthermore, while the critical height is adjacent to the wave surface in both case A1 and A2, it lifts at approximately $\theta=30^{\circ}$, and

\footnotetext{
${ }^{1}$ Due to a difference in the coordinate system applied, there is a sign change in the vorticity when comparing the results of this study with the study of Buckley \& Veron (2016)
} 


\begin{tabular}{|c|c|c|c|c|c|c|c|c|c|c|c|c|c|c|}
\hline \multirow{2}{*}{ Avg. case } & \multirow{2}{*}{$U_{s l}$} & \multirow{2}{*}{$U_{s g}$} & \multicolumn{3}{|c|}{ Q1 } & \multicolumn{3}{|c|}{ Q2 } & \multicolumn{3}{|c|}{ Q3 } & \multicolumn{3}{|c|}{ Q4 } \\
\hline & & & $\overline{\epsilon_{0}}$ & $\alpha$ & $N_{a v}$ & $\epsilon_{0}$ & $\alpha$ & $N_{a v}$ & $\epsilon_{0}$ & $\alpha$ & $N_{a v}$ & $\epsilon_{0}$ & $\alpha$ & $N_{a v}$ \\
\hline A1 - & 0.1 & 1.5 & 0.09 & $8 \%$ & 46 & 0.09 & $10 \%$ & 49 & -0.08 & $8 \%$ & 47 & -0.08 & $7 \%$ & 47 \\
\hline A2 - & 0.1 & 1.5 & 0.16 & $17 \%$ & 42 & 0.14 & $17 \%$ & 43 & -0.12 & $20 \%$ & 40 & -0.13 & $20 \%$ & 40 \\
\hline B1 - median $\epsilon$ & 0.1 & 2.1 & 0.14 & $11 \%$ & 46 & 0.17 & $10 \%$ & 46 & -0.09 & $10 \%$ & 44 & -0.09 & $14 \%$ & 43 \\
\hline
\end{tabular}

Table 2: Overview of three averaging cases. Quadrant observations with steepness within $\epsilon_{0} \pm \alpha \epsilon_{0}$ used in averaging. $N_{a v}$ : number of wave quadrants within the selected limits.

\begin{tabular}{|c|c|c|c|c|c|c|c|c|c|}
\hline Avg. Case & $U_{s l}$ & $U_{s g}$ & $\bar{\eta}_{c}[\mathrm{~mm}]$ & $\bar{\eta}_{t}[\mathrm{~mm}]$ & $\bar{\lambda}_{q, 1}[\mathrm{~mm}]$ & $\bar{\lambda}_{q, 2}[\mathrm{~mm}]$ & $\bar{\lambda}_{q, 3}[\mathrm{~mm}]$ & $\bar{\lambda}_{q, 4}[\mathrm{~mm}]$ & $a k$ \\
\hline A1 - median $\epsilon$ & 0.1 & 1.5 & 2.0 & -1.8 & 22.8 & 23.2 & 22.9 & 22.4 & 0.13 \\
\hline A2 - max $\epsilon$ & 0.1 & 1.5 & 2.6 & -2.5 & 17.1 & 18.1 & 19.6 & 18.3 & 0.22 \\
\hline B1 - median $\epsilon$ & 0.1 & 2.1 & 5.5 & -3.4 & 39.7 & 30.8 & 38.7 & 36.8 & 0.19 \\
\hline
\end{tabular}

Table 3: Mean crest and trough elevation $\left(\bar{\eta}_{t}, \bar{\eta}_{c}\right)$, and mean length $\bar{\lambda}_{q}$ of each quadrant for averaging cases considered. $a k=\pi\left(\left|\bar{\eta}_{c}\right|+\left|\bar{\eta}_{t}\right|\right) /\left(\bar{\lambda}_{q, 1}+\right.$ $\left.\bar{\lambda}_{q, 2}+\bar{\lambda}_{q, 3}+\bar{\lambda}_{q, 4}\right)$

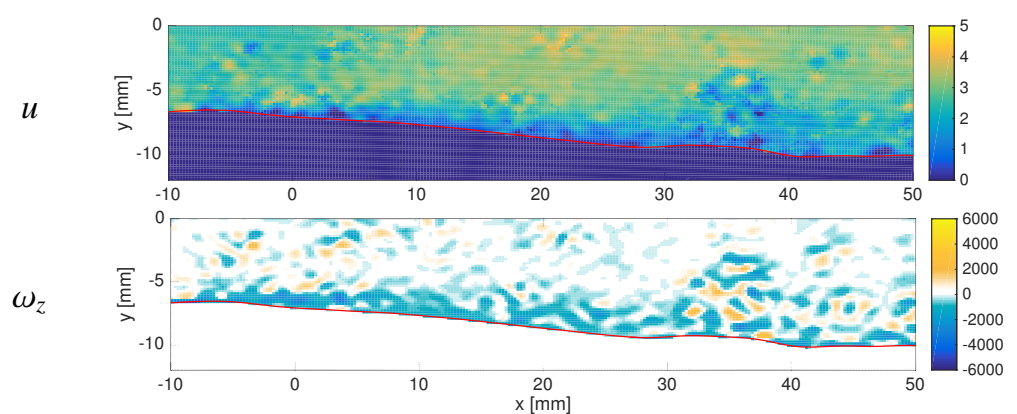

a) $U_{s g} 1.50 \mathrm{~m} / \mathrm{s}, \epsilon_{Q 2} \approx 0.08($ Case $\mathrm{A} 1)$

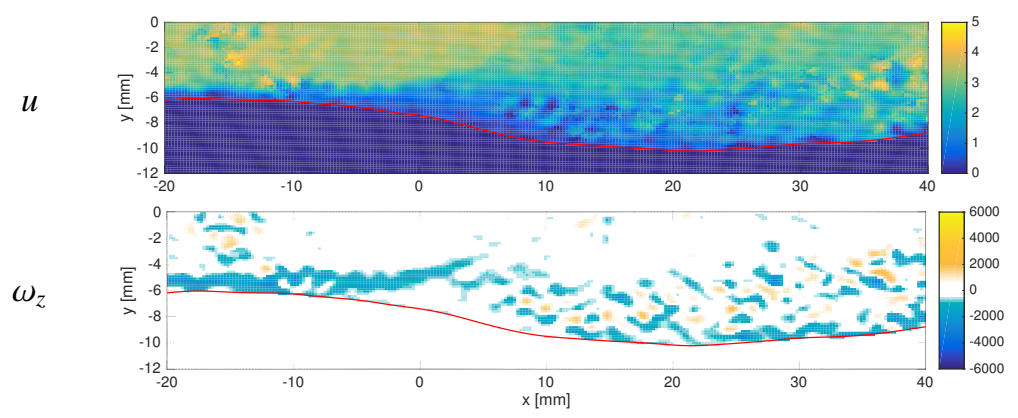

b) $U_{s g} 1.50 \mathrm{~m} / \mathrm{s}, \epsilon_{Q 2} \approx 0.14($ Case A2)

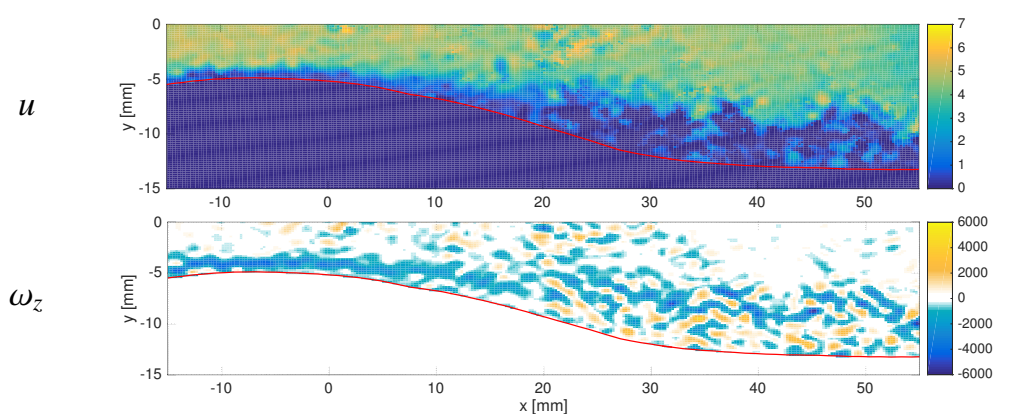

c) $U_{s g} 2.10 \mathrm{~m} / \mathrm{s}, \epsilon_{Q 2} \approx 0.16($ Case B1)

Figure 7: Contour plot of horizontal velocity $[\mathrm{m} / \mathrm{s}]$ (top) and spanwise vorticity $\left[\mathrm{s}^{-1}\right]$ (bottom) for three instantaneous PIV velocity fields. Representative flow field for the three averaging cases: a) median steepness $U_{s g} 1.50 \mathrm{~m} / \mathrm{s}$ (case A1), b) maximum steepness $U_{s g} 1.50 \mathrm{~m} / \mathrm{s}$ case (case A2), c) median steepness $U_{s g} 2.10 \mathrm{~m} / \mathrm{s}$ (case B1).

reattaches at approximately $\theta=-50^{\circ}$ in case B1. Note that the critical height is based on the characteristic wave speed $c$ for each experimental case (ref. section 2.4). While some variations in the wave speed was observed 
$\langle u\rangle$

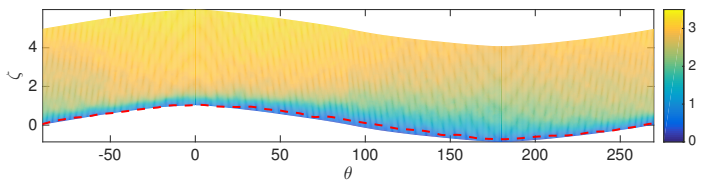

$\langle v\rangle$

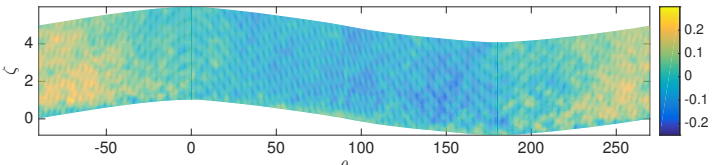

$\bar{u}$

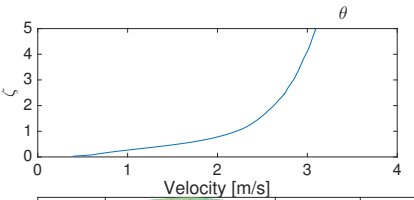

$\tilde{u}$

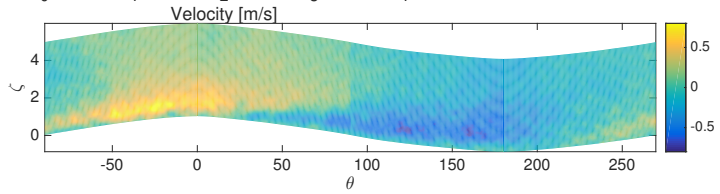

a) $U_{s g} 1.50 \mathrm{~m} / \mathrm{s}, a k=0.13$ (case A1)
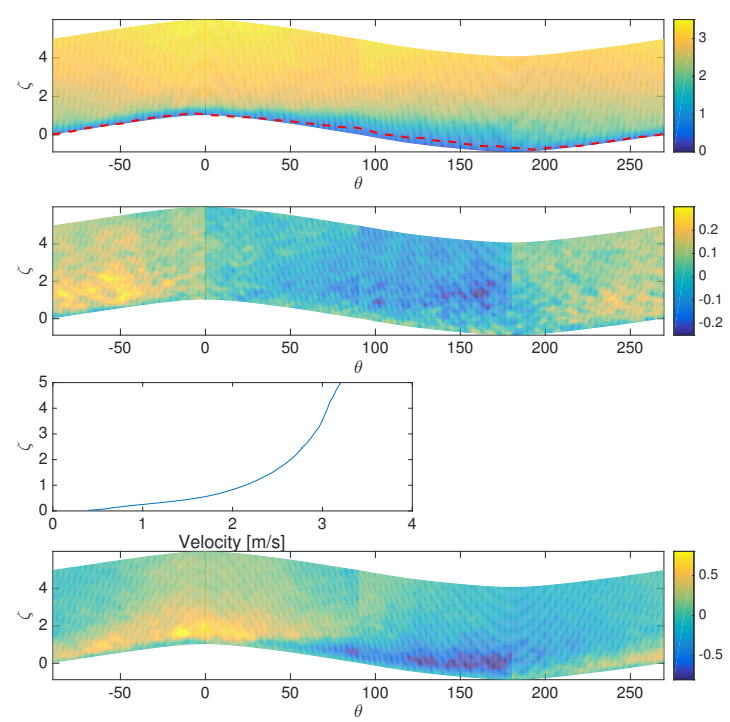

b) $U_{s g} 1.50 \mathrm{~m} / \mathrm{s}, a k=0.22$ (case A2)

$\langle u\rangle$

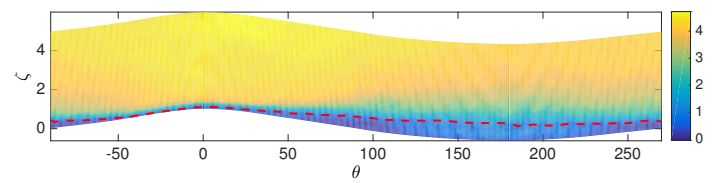

$\langle v\rangle$
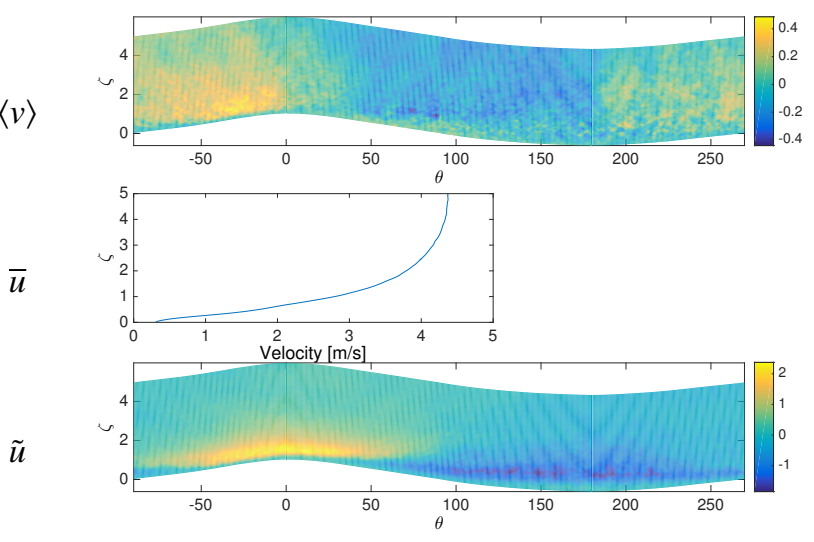

c) $U_{s g} 2.10 \mathrm{~m} / \mathrm{s}, a k=0.19$ (case B1)

Figure 8: Mean flow structure of the three averaging cases investigated. All velocities are in $[\mathrm{m} / \mathrm{s}]$. Red-dashed line of the $\langle u\rangle$ plot indicates the location of the critical height $z_{i}$

considering the cross correlation of individual wave components, these were relatively small, and selecting a different characteristic wave speed within the range observed will not qualitatively change the evolution of the critical height plotted in figure 8 .

The wave-coherent mode of case B1 contains, in its peak, almost $50 \%$ of the mean flow velocity. The wavecoherent velocities are directly related to the low frequency peak fluctuations measured by the hot-wire probes presented by Ayati et al. (2016).

Note that while case A1 and B1 provide relatively smooth transitions between the four wave quadrants, case A2 exhibits more abrupt transitions. This indicates that the steepness combination chosen for case A2 is not char- acteristic of the waves in the system. While the averaging illustrates how the (high) steepness affects the flow in each individual quadrant, the combination of the four quadrants presented in figure $8 \mathrm{~b}$ ) does not necessarily represent the typical flow over steep waves in the system. For these reasons, we will focus on the cases A1 and B1 in the following.

When comparing cases $\mathrm{A} 1 / \mathrm{A} 2$ and $\mathrm{B} 1$, it should be recalled that as the vertical coordinate $\zeta$ is normalized by the crest amplitude, the maximum $\zeta$ value in case B1 represents a larger physical distance from the interface compared with case A1/A2. In fact, $\zeta=5$ in case B1 is approximately midway between the interface and upper wall. For this reason, the mean velocity profile in case B1 
reaches its centerline value at $\zeta=5$, while this does not happen for cases A1 and A2. Nonetheless, the mean velocity profiles provide valuable information concerning the boundary layer, i.e. the region with strong velocity gradient. It stretches up to $\zeta \approx 1$ in cases $\mathrm{A} 1$ and $\mathrm{A} 2$ and to $\zeta \approx 2$ in case B1. This indicates that large amplitude waves induce a thicker boundary layer, i.e., momentum is pushed away from the interface. This effect is related to the wave induced stress $-\tilde{u} \tilde{v}$. The results of the phase averaged velocities presented in figure 8 are qualitatively in agreement with the results presented by Buckley \& Veron (2016) for airflow over slow waves in an open system. As discussed by Buckley \& Veron, the airflow pattern gives rise to a negative wave induced momentum flux, transporting horizontal momentum away from the interface.

In figure 9, the streamlines of the phase-averaged velocity field, seen in a frame of reference moving with the representative wave speed, is plotted for cases A1 and B1. The streamlines are coloured with the kinetic energy of the phase averaged flow field $\left(0.5\left(\langle u\rangle^{2}+\langle v\rangle^{2}\right)\right.$.

In case $\mathrm{A} 1$, the streamlines closely follow the average wave profile, except near the critical layer, where two small regions of closed streamlines can be seen at $\theta=100^{\circ}$ and $180^{\circ}$.

In case B1, a distinct cat's eye (Lighthill, 1962) is observed slightly downstream of the crest $\left(\theta=50^{\circ}\right)$. The position of the cat's eye implies that on average the waves experience a co-moving pocket of air located slightly downwind of the crest. The streamlines adjacent to crest are pushed away from the wave surface and the shape of the streamlines above the cat's eye structure are shifted approximately $40^{\circ}$ compared with the wave profile. This asymmetry of the flow field is believed to produce an asymmetric pressure distribution along the wave.

The results for case B1 are qualitatively similar to the LES results by Hara \& Sullivan (2015), investigating a linear wave in open channel flow under strong wind forcing, with $c / u_{*}=1.6$ and wave steepness $a k=0.226$. As demonstrated by Hara \& Sullivan, such an asymmetry in the critical layer causes the peak pressure to move downstream from the wave trough to the Q4 region. Here the high pressure pushes on the positive surface slope and contributes to the air-water momentum flux, pushing the wave in the flow direction. This effect is related to the sheltering effect by Jeffreys (1925), and the nonseparated sheltering by Belcher \& Hunt (1998). Although there are differences in an open air-sea system and a closed closed pipe section, a similar effect is expected to apply in our experimental cases, and particularly in case B1 where a significant lifting of the critical height and asymmetry between the wave shape and streamlines above the wave is observed. The distribution of the mean flow kinetic energy (indicated by the colouring of the streamlines in figure 9) indicate that this is the case, as the horizontal position of the peak kinetic energy (associated with lowpressure zone) is seen to be located on the leeward side of the crest for case B1.

\subsection{Vortical structures}

The distribution of vortical structures gives insight into the turbulence of the flow, being one of the main features of turbulent wall-bounded flow (Chen et al., 2014). The swirling strength criterion proposed by Adrian et al. (2000) is employed to identify vortical motion on the 2D PIV plane. The criterion discriminates regions of swirling motion from regions of shear, ensuring that coherent regions of swirling strength will contain rotational flow structures. A directional swirling strength is given as follows (Wu \& Christensen, 2006):

$$
\Lambda_{c i}=\lambda_{c i} \frac{\omega_{z}(x, y)}{\left|\omega_{z}(x, y)\right|},
$$

where $\lambda_{c i}$ is the imaginary part of the complex eigenvalue of the velocity-gradient tensor and $\omega_{z}(x, y)$ is the spanwise vorticity. $\Lambda_{c i}$ will be referred to as the swirling strength in the remainder of the paper. Using this definition, vortical structures with clockwise rotation will have a negative swirling strength, while vortical structures with an anti-clockwise rotation will have a positive swirling strength. Furthermore, the sign-dependent normalization scheme proposed by Chen et al. (2014) is applied to remove the wall-normal dependence of the swirling strength

$$
\Lambda_{n}= \begin{cases}\frac{\Lambda_{c i}(x, y)}{\Lambda_{\text {arg }}^{+}(y)} & \text { if } \Lambda_{c i}>0 \\ \frac{\Lambda_{c i}(x, y)}{\left|\Lambda_{\text {avg }}^{-}(y)\right|} & \text { if } \Lambda_{c i}<0,\end{cases}
$$

where $\Lambda_{a v g}^{+}(y)=\left\langle\Lambda_{c i}(y) \mid \Lambda_{c i}(y)>0\right\rangle$ and $\Lambda_{a v g}^{-}(y)=$ $\left\langle\Lambda_{c i}(y) \mid \Lambda_{c i}(y)<0\right\rangle . \Lambda_{n}$ is referred to as the normalized swirling strength.

As the flow is bounded by two boundaries (upper pipe wall and water interface), the normalization in equation 3 is modified slightly. Separate $\Lambda_{\text {avg }}^{+}(y)$ and $\Lambda_{\text {avg }}^{-}(y)$ are calculated for the region above and below the air center (midpoint between the mean interface and upper pipe wall). Above the air center $y$ is the distance to the stationary upper wall, and below the air center $y$ is the distance to the interface. $\Lambda_{a v g}^{+}$and $\Lambda_{a v g}^{-}$was evaluated for all available velocity fields (independent of wave quadrant dependency) for the two experimental cases investigated. Results are presented in figure 10. The profiles of $\Lambda_{a v g}^{+}$ and $\Lambda_{\text {avg }}^{-}$are seen to be continuous across the mean air centreline.

As the velocity gradient near the upper wall is larger than near the moving interface, the characteristic strength of vortical structures near the upper wall is higher than at the interface (see figure 10). Case A exhibits a narrow region of energetic structures close to the interface, resembling the (inverse) profile at the upper wall. Meanwhile, case B exhibits a thicker layer of strong vortices near the interface. This indicates that while the airflow in case A behaves approximately as the flow over a flat wall, vortical structures are considerably affected by the larger waves in case $\mathrm{B}$. 


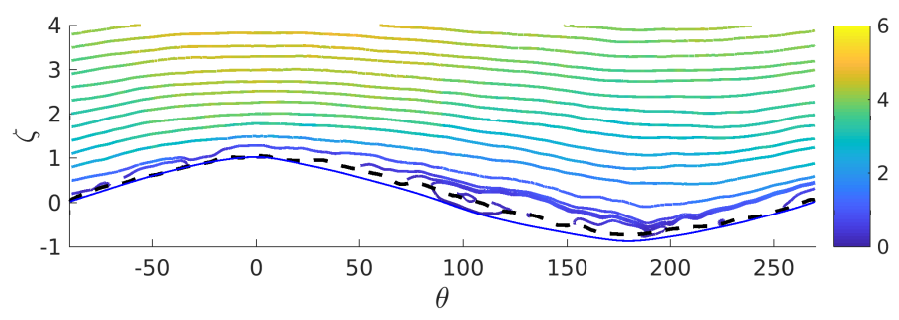

a) $U_{s g} 1.50 \mathrm{~m} / \mathrm{s}, a k=0.13$ (case $\left.\mathrm{A} 1\right)$

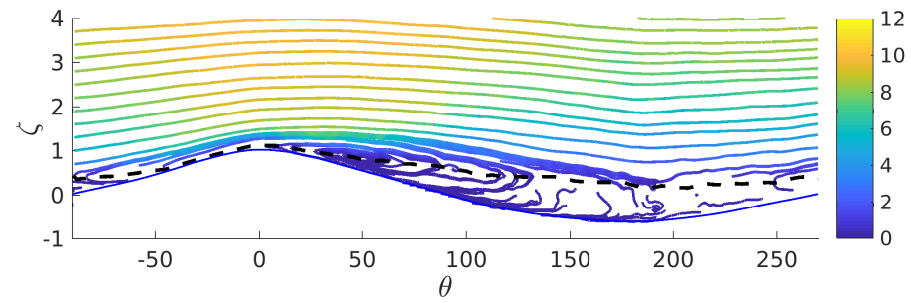

b) $U_{s g} 2.10 \mathrm{~m} / \mathrm{s}, a k=0.19($ case B1)

Figure 9: Streamlines of $\boldsymbol{u}-c$ for averaged flow field. Colorbar represents kinetic energy of the mean flow divided by fluid density $\left(0.5\left(\langle u\rangle^{2}+\langle v\rangle^{2}\right)\right.$ $\left[\mathrm{m}^{2} / \mathrm{s}^{2}\right]$. Dashed line indicates the position of the critical layer.

In order to extract coherent regions of swirling motion, a non-zero threshold of $\left|\Lambda_{n}\right|$ has to be used. Chen et al. (2014) used a threshold of $\left|\Lambda_{n}\right|>1$, this is also applied here. To reduce the probability of including vortical structures generated by errors in the PIV, a minimum of three vectors across is required for a region to constitute a vortical structure.

When evaluating the swirling strength, a 7x7 finite difference filter was applied. The filter acts to smooth out the calculated swirling strength field and small PIV errors. The filter also dictates the size of structures to be extracted, as a larger filter will identify larger regions of swirling motion, and hence extract larger structures. The filter size was chosen to ensure that the vortical structures extracted had a single vortex core, when a Galilean decomposition of the local velocity field was applied (see section 3.3.1). As we are mostly interested in the distribution of vortical structures in the crest-to-trough region, it was necessary to select a filter size able to extract structures smaller than the wave amplitude.

\subsubsection{Instantaneous swirling events}

In figure 11 instantaneous vector plots of the individual swirling events (coherent regions with $\left|\Lambda_{n}\right|>1$ ) are plotted using a local Galilean decomposition of the velocity field. The normalization of the swirling strength ensures that vortical structures are identified throughout the pipe cross section, but the structures close to the interface and upper pipe wall are in general more energetic than at the pipe centre. Note that the region of the pipe from $y=20$ to $30 \mathrm{~mm}$ is heavily influenced by local light reflections, hence this region is omitted from figure 10 and should not be trusted in figure 11 .

Figure 11 reveals a significant number of rotating structures of the flow. From the close-up section of figure 11 (c and d) it can be seen that the identified structures exhibit a rotating motion, when a local Galilean decomposition of the velocity field is used. Close to the top wall, positive vortices dominate. This is to be expected as the flow is directed from left to right in the plots. Close to the interfaces the identified vortices are small, ranging from approximately 1 to $3 \mathrm{~mm}$ in diameter. Away from the interfaces some larger (up to $6 \mathrm{~mm}$ diameter) regions of positive (above the air center) and negative (below the air center region) swirling events are observed. As a cut-off value $\left(\left|\Lambda_{n}\right|>1\right)$ is used, these size estimates are not absolute, but relate to the size of the high energy core of the vortex.

In case A (figure 11 a) the region above the interface is mainly populated by negative vortices. This is analogous to the vortices observed close to the top wall. For case B, positive vortices are observed close to the interface in the crest-to-trough region (figure $11 \mathrm{~b}$ ). From the crest and along the streamwise direction, a number of negative swirling events are observed, suggesting that vortical structures on the windward side of the crest are shed locally. A similar observation was made by Ayati et al. (2016), analyzing instantaneous velocity fields like the ones presented in figure 11. More evidence of this behavior is seen in the next section.

\subsubsection{Phase dependency of swirling events}

During the phase-averaging procedure, the normalized peak strength and location of each identified swirling event was registered. Scatter plots of all swirling events for cases A1 and B1 are presented in figure $12 \mathrm{a}$ ) and b). In the middle part of figure $12 \mathrm{a}$ ) and $\mathrm{b}$ ), the average number of swirling events within $0 \leq \zeta \leq 1$ and $0 \leq \zeta \leq 3$ is plotted as a function of wave phase. At the bottom, the average peak $\Lambda_{n}$ within these intervals is plotted, also as a function of wave phase.

In case A1, the distribution of swirling events (figure 

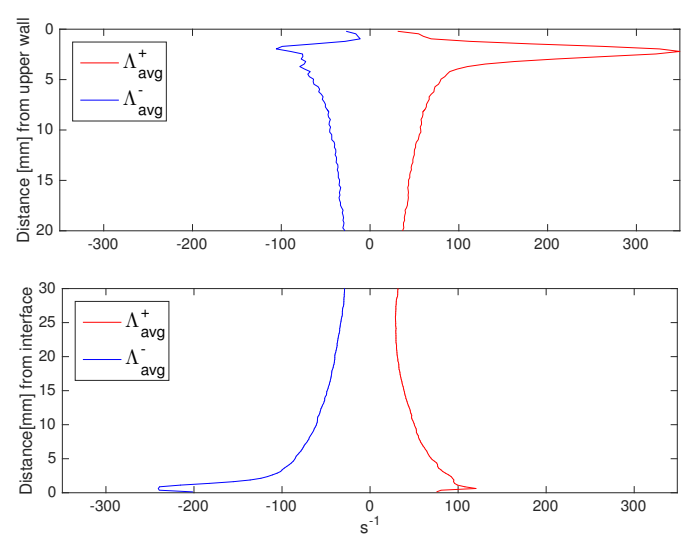

a) $U_{s g} 1.50 \mathrm{~m} / \mathrm{s}$ (case A).
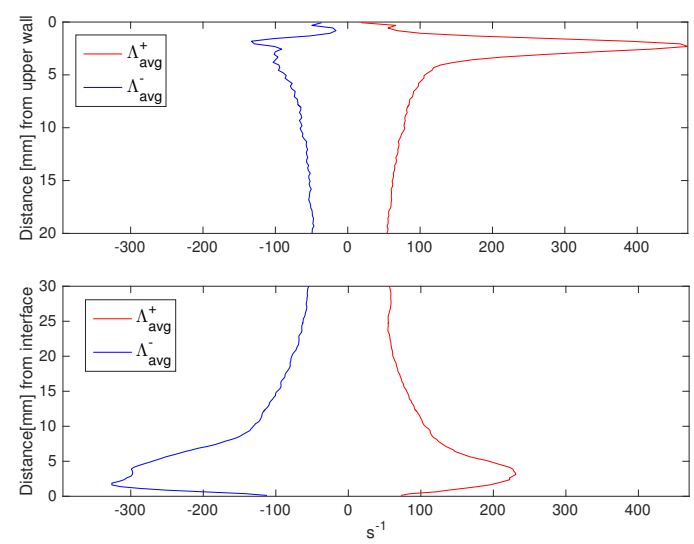

b) $U_{s g} 2.10 \mathrm{~m} / \mathrm{s}$ (case B).

Figure 10: $\Lambda_{\text {avg }}^{+}$and $\Lambda_{\text {avg }}^{-}\left[s^{-1}\right]$ evaluated above (top) and below (bottom) the mean air centerline for the two experimental cases. a) $U_{s g} 1.50 \mathrm{~m} / \mathrm{s}$, and b) $U_{s g} 2.10 \mathrm{~m} / \mathrm{s}$.

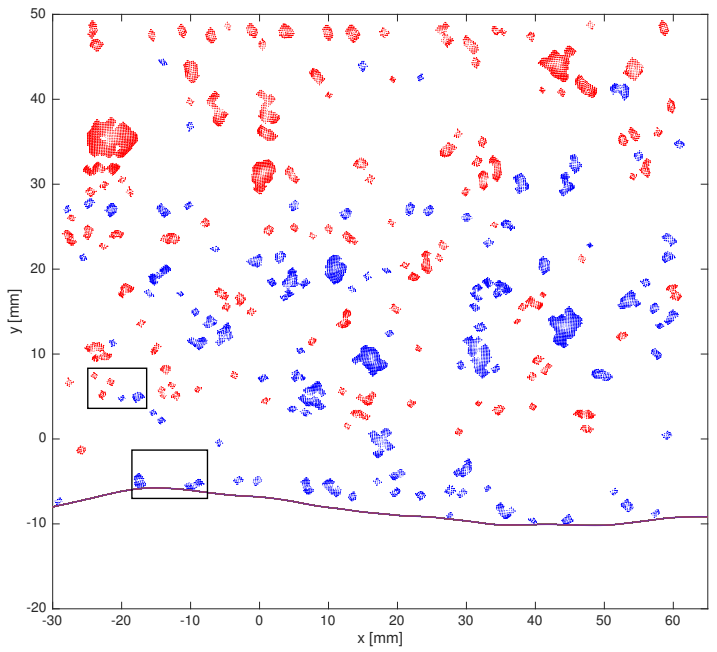

a) $U_{s g}=1.50 \mathrm{~m} / \mathrm{s}($ Case A), wavy surface

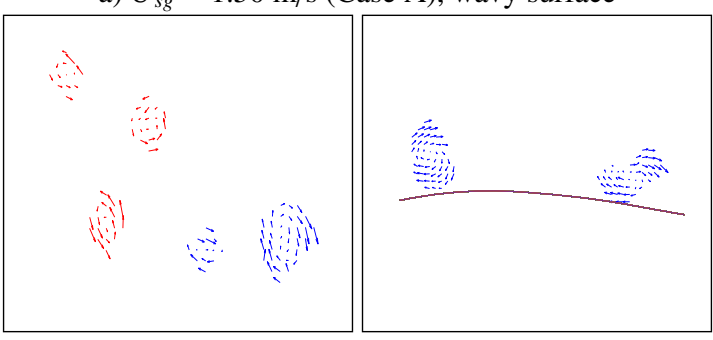

c) $U_{s g}=1.50 \mathrm{~m} / \mathrm{s}($ case A), Close up

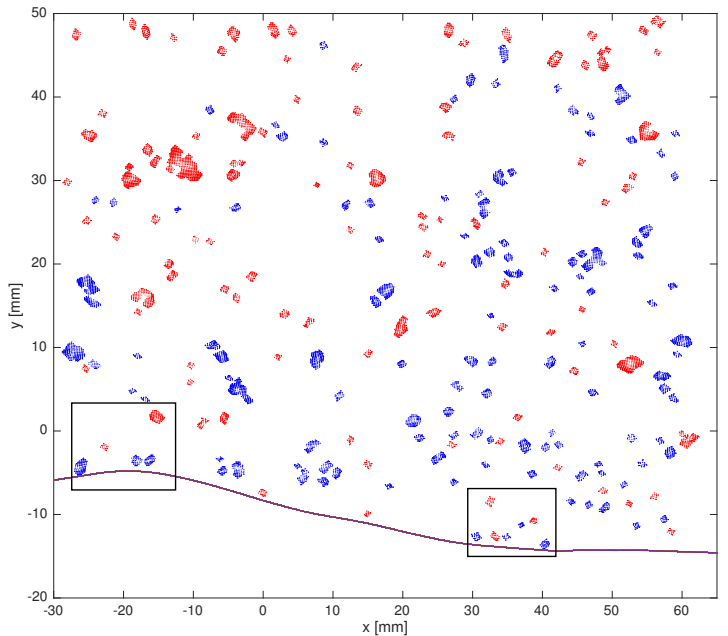

b) $U_{s g}=2.10 \mathrm{~m} / \mathrm{s}$ (case B), wavy surface

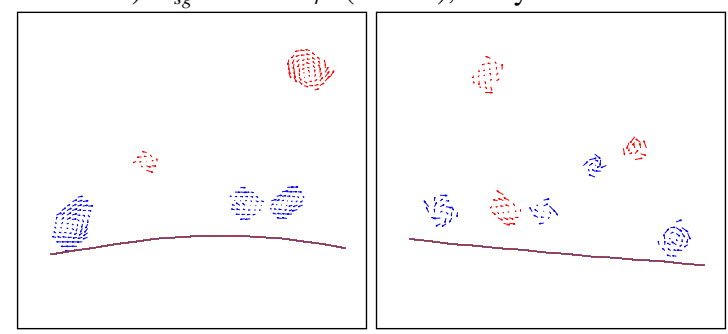

d) $U_{s g}=2.10 \mathrm{~m} / \mathrm{s}$ (case B), close up

Figure 11: Local Galilean decomposition of the instantaneous flow field showing vector plots of each identified swirling event. Positive (counterclockwise rotation) swirling events coloured in red. Negative (clockwise rotation) in blue. Flow direction from left to right. a-b) Instantaneous vortices for case A (a) and case B (b). c,d) Close up of vortical structures in figure a and b (location indicated by black box in a and b).

12 a) reveals only a weak phase dependence. Close to the interface $(0 \leq \zeta \leq 1)$, there are slightly more negative swirling events above the crest than on the leeward side of the wave. Note that the mean strength of positive vortices within $0 \leq \zeta \leq 1$ is not included in figure 12 a) since no positive swirling events were observed here. In general, case A1 exhibits a weak phase dependency in both registered swirling events and phase-averaged swirling strength, indicating that the waves do not significantly alter the distribution of vortical structures.

In case B 1, a clear phase dependence of swirling events is observed (figure $12 \mathrm{~b}$ ). Close to the interface $(0 \leq$ $\zeta \leq 1$ ), a large concentration of negative swirling events is observed on the windward side of the wave, between the reattachment point of the critical layer to the crest $\left(-50^{\circ}<\theta<0^{\circ}\right)$. These vortices are then shed from 

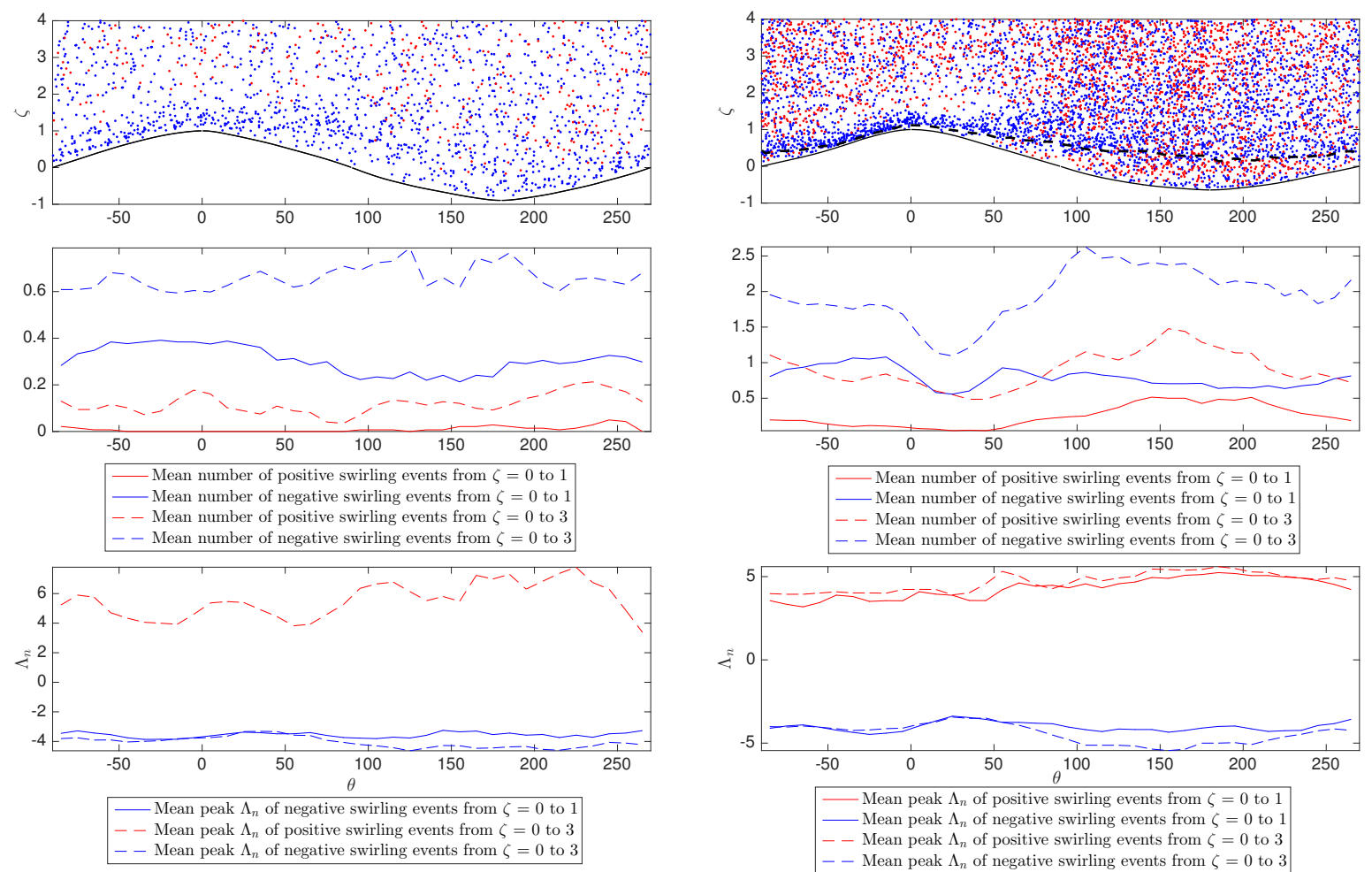

a) $U_{s g}=1.50 \mathrm{~m} / \mathrm{s},($ case A1)

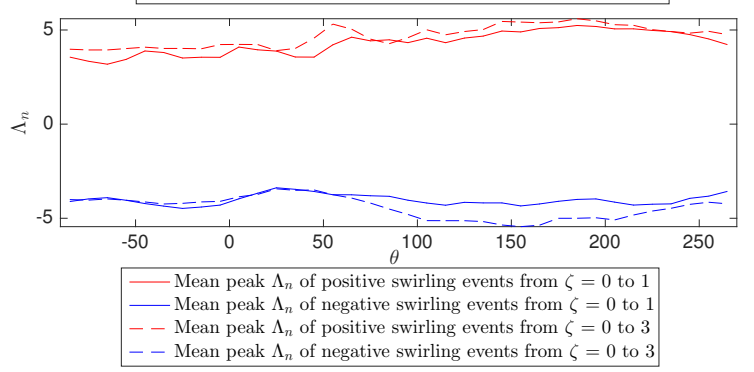

b) $U_{s g}=2.10 \mathrm{~m} / \mathrm{s}$, (case B1)

Figure 12: Phase dependency of swirling events for the two median steepness cases. Top: Scatter plot of all positive (red) and negative (blue) swirling events. Black dotted line represents critical height. Middle: Mean number of positive (red) and negative (blue) swirling events $\left(N_{s w} /\left(N_{q} \Delta \theta\right)\right)$ from $\zeta$ $=0$ to 1 (full line) and from $\zeta=0$ to 3 (dashed line). Bottom: Mean peak normalized swirling strength of positive (red) and negative (blue) swirling structures detected from $\zeta=0$ to 1 (full line) and from $\zeta=0$ to 3 (dashed line).

the crest of the wave. Slightly downstream at $\theta=50^{\circ}$, the number of positive swirling events close to the interface increases, and reaches a maximum above the trough. These observations are further elucidated by the middle plot of figure $12 \mathrm{~b}$ ), which clearly shows that there is a considerably higher concentration of vortices where the critical layer is lifted from the surface. The total number of swirling events (positive and negative combined) is maximum above the trough, indicating a highly turbulent region, characteristic of a separated shear layer (Buckley \& Veron, 2016).

The bottom plot of figure $12 \mathrm{~b})$ shows that although the variations in mean peak strength $\left(\Lambda_{n}\right)$ are more subtle than the variation in number of swirling events, the vortices (both positive and negative) are more energetic above the trough.

The phase-averaged vorticity field $\left\langle\omega_{z}\right\rangle$ is plotted for all three averaging cases in figure 13 . The vorticity field is seen to follow the critical height in case B1, and the criterion for shear layer separation is fulfilled for the phaseaveraged vorticity field (ref. section 3.1). Below the critical height is a region dominated by positive vorticity. This is related to the high concentration of positive swirling events observed below the critical height (ref. figure $12 \mathrm{~b}$ ).
It is interesting to note that shear layer separation is observed for case B1, as this experimental case is in the regime of "amplitude saturation" (ref. section 2.4). As discussed in section 3.1, airflow separation above waves is traditionally linked to the onset of wave breaking, and while new experimental (Buckley \& Veron, 2016) and numerical (Sullivan et al., 2018) results indicate that separation may occur for waves without active wave breaking, the shear layer separation observed in case B1 is assessed to be a strong indication of dissipative mechanisms in the waves (microbreaking or spilling) which may help explain the amplitude saturation observed in previous studies (Ayati et al., 2015).

In both $\mathrm{A} 1$ and $\mathrm{A} 2$ the high intensity vorticity field remains adjacent to the wave surface. Based on the instantaneous vorticity plots presented in figure 7 this is to be expected for A1, but for A2 the instantaneous vorticity field (figure $7 \mathrm{~b}$ ) indicated a slight separation behind the crest. While a clear separation like the results observed for case B1 are not observed in either case A1 or A2, there is a marked reduction in the shear layer intensity along the third quadrant of the wave, and the phase averaged vorticity field is observed to extend further away from the interface in this region, indicating that intermittent airflow separation occurs also for these two cases, 


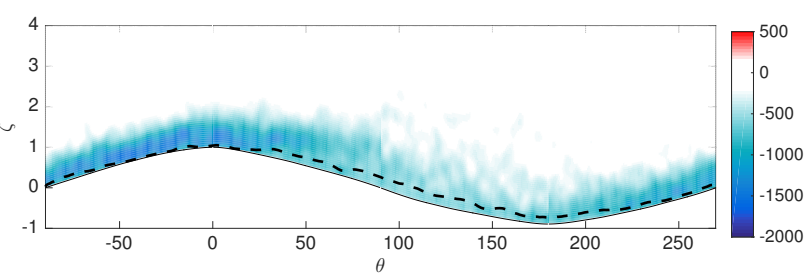

a) $U_{s g}=1.50 \mathrm{~m} / \mathrm{s}, a k=0.13($ case A1)

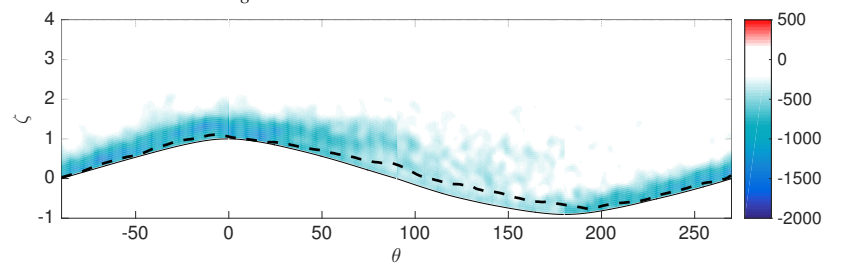

b) $U_{s g}=1.50 \mathrm{~m} / \mathrm{s}, a k=0.22($ case A2)

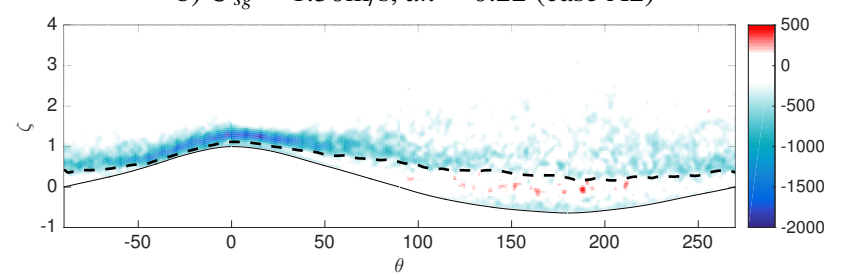

c) $U_{s g}=2.10 \mathrm{~m} / \mathrm{s}, a k=0.19($ case B1)

Figure 13: Phase averaged vorticity field $\left(\left\langle\omega_{z}\right\rangle\right)$ for the three averaging cases under investigation

but that the frequency of occurrence is significantly lower than for case B1.

\section{Concluding remarks}

We present an experimental investigation of air-water flow in a horizontal pipe. Two experimental cases with $U_{s l}=0.1 \mathrm{~m} / \mathrm{s}$ are investigated, increasing $U_{s g}$ from 1.5 to $2.1 \mathrm{~m} / \mathrm{s}$. The first case contains small, moderate-steepness waves $\left(\eta_{r m s}=1.25 \mathrm{~mm}, a k \sim 0.13\right.$ (median steepness)), whilst the second case contains higher and steeper waves $\left(\eta_{r m s}=3.00 \mathrm{~mm}, a k \sim 0.19\right.$ (median steepness)).

The conditional phase-averaging procedure reveals that the velocity field at $U_{s g}=2.10 \mathrm{~m} / \mathrm{s}$ displays a thicker region of sheltered airflow on the leeward side of the crest, compared with the flow at $U_{s g}=1.50 \mathrm{~m} / \mathrm{s}$. In this region the critical layer is seen to lift from the interface for the $U_{s g}=2.10 \mathrm{~m} / \mathrm{s}$ case. In a wave co-moving frame of reference, the phase-averaged streamlines form a closed pattern (cat's eye) around the critical height and slightly downwind of the crest. This implies that the typical wave crest has a pocket of circulating air in its front. This is assessed to impact on the pressure field above the waves, resulting in increased form drag along the wave profile.

The analysis of small scale vortical structures and the evaluation of the average shear layer along the wave reveals significant differences between the two experimental cases. At $U_{s g}=1.50 \mathrm{~m} / \mathrm{s}$ (considering both median and maximum wave steepness), we observe a shear layer dominated by negative vorticity that remains adjacent to the wave surface along the entire wave profile. At
$U_{s g}=2.10 \mathrm{~m} / \mathrm{s}$, this layer detaches from the surface just downstream of the crest. The swirling strength criterion reveals that below the detached layer of negative vorticity, there is a region dominated by opposite-signed vortices. The border between these regions coincides with the critical height.

Airflow separation is known to alter the wind-to-wave momentum transfer, and while further analysis is needed to quantify the impact of separation on the momentum transfer (and wave-growth), it is interesting to note that the $U_{s g}=2.10 \mathrm{~m} / \mathrm{s}$ case is in the region of amplitude saturation (Ayati et al., 2015). Future studies will investigate whether the onset of separation is related to the amplitude saturation observed by Ayati et al. for superficial gas velocities above $1.75 \mathrm{~m} / \mathrm{s}$. To assess this question it will be necessary to obtain an improved understanding of how airflow separation, possible wave breaking and other dissipative mechanisms interact, considering the closed geometry and pressure driven flow which is characteristic of the two-phase pipe flow. This is the subject of current investigations.

\section{Acknowledgement}

The authors wish to acknowledge the strategic research initiative EarthFlows and the Academia program at the Faculty of Mathematics and Natural Sciences at the University of Oslo. Thanks to Jostein Kolaas for help with the HydroLab PIV code. Laboratory Head Engineer Olav Gundersen is gratefully acknowledged for the technical assistance he provided for the experimental work. 


\section{References}

Adrian, R. J., Christensen, K. T. \& Liu, Z. C. 2000 Analysis and interpretation of instantaneous turbulent velocity fields. Experiments in fluids 29 (3), 275-290.

AndRItsos, N. \& HanRatTy, T. J. 1987 Influence of interfacial waves in stratified gas-liquid flows. AIChE Journal 33, 444-454.

Ayati, A.A., Kolaas, J., Jensen, A. \& Johnson, G.W. 2014 A PIV investigation of stratified gas-liquid flow in a horizontal pipe. International Journal of Multiphase Flow 61, 129 - 143.

Ayati, A.A., Kolaas, J., Jensen, A. \& Johnson, G.W. 2015 Combined simultaneous two-phase PIV and interface elevation measurements in stratified gas-liquid pipe flow. International Journal of Multiphase Flow 74, 45 - 58.

Ayati, A.A., Kolaas, J., Jensen, A. \& Johnson, G.W. 2016 The effect of interfacial waves on the turbulence structure of stratified air-water pipe flow. International Journal of Multiphase Flow 78, $104-116$.

Ayati, A. A. \& Carneiro, J. N. E. 2018 Statistical characterization of interfacial waves in turbulent stratified gas-liquid pipe flows. International Journal of Multiphase Flow 103, 94-105.

Ayati, A. A., Farias, P. S. C., Azevedo, L. F. A. \& de Paula, I. B. 2017 Characterization of linear interfacial waves in a turbulent gas-liquid pipe flow. Physics of Fluids 29 (6), 062106.

Ayati, A. A., Vollestad, P. \& Jensen, A. 2018 Detailed measurements of interfacial dynamics in air-water pipe flow. Procedia IUTAM 26 , 59-69.

Banner, M. L \& Melville, W. K. 1976 On the separation of air flow over water waves. Journal of Fluid Mechanics 77 (04), 825-842.

Banner, M. L. \& Peirson, W. L. 1998 Tangential stress beneath winddriven air-water interfaces. Journal of Fluid Mechanics 364, 115145 .

Barmak, I., Gelfgat, A., Vitoshin, H., Ullmann, A. \& Brauner, N. 2016 Stability of stratified two-phase flows in horizontal channels. Physics of Fluids 28 (4), 044101.

BarneA, D. \& TAITEL, Y. 1993 Kelvin-helmholtz stability criteria for stratified flow: viscous versus non-viscous (inviscid) approaches. International journal of multiphase flow 19 (4), 639-649.

Belcher, S. E. \& Hunt, J. C. R. 1998 Turbulent flow over hills and waves. Annual Review of Fluid Mechanics 30 (1), 507-538.

Bendiksen, K. \& EsPedal, M. 1992 Onset of slugging in horizontal gasliquid pipe flow. International journal of multiphase flow 18 (2), 237-247.

BIBERG, D. 2007 A mathematical model for two-phase stratified turbulent duct flow. Multiphase Science and Technology 19, 1-48.

Birvalski, M., Tummers, M. J., Delfos, R. \& Henkes, R.A.W.M. 2014 PIV measurements of waves and turbulence in stratified horizontal two-phase pipe flow. International Journal of Multiphase Flow 62 , 161-173.

Birvalski, M., Tummers, M. J., Delfos, R. \& Henkes, R. A. W. M. 2015 Laminar-turbulent transition and wave-turbulence interaction in stratified horizontal two-phase pipe flow. Journal of Fluid Mechanics 780, 439456

BuckLey, M. P. \& VERON, F. 2016 Structure of the airflow above surface waves. Journal of Physical Oceanography 46 (5), 1377-1397.

Campbell, B. K., Hendrickson, K. \& Liu, Y. 2016 Nonlinear coupling of interfacial instabilities with resonant wave interactions in horizontal two-fluid plane couettepoiseuille flows: numerical and physical observations. Journal of Fluid Mechanics 809, 438479.

Campbell, B. K. \& Liu, Y. 2016 A nonlinear flow-transition criterion for the onset of slugging in horizontal channels and pipes. Physics of Fluids $\mathbf{2 8}$ (8), 082103.

Chen, Q., Zhong, Q., Wang, X. \& LI, D. 2014 An improved swirlingstrength criterion for identifying spanwise vortices in wall turbulence. Journal of Turbulence 15 (2), 71-87.

Deike, L, Fuster, D, Berhanu, M \& Falcon, E 2014 Direct numerical simulations of capillary wave turbulence. Physical review letters 112 (23), 234501.

Fernandino, M. \& Ytrehus, T. 2006 Determination of flow sub-regimes in stratified air-water channel flow using LDV spectra. I.J. Multiphase Flow 32, 436-446.

Gent, P. R. \& TAYlor, P. A. 1977 A note on separation over short wind waves. Boundary-Layer Meteorology 11 (1), 65-87.
Grare, L., Peirson, W. L., Branger, H., Walker, J. W., Giovanangeli, J. P. \& MaKin, V. 2013 Growth and dissipation of wind-forced, deepwater waves. Journal of Fluid Mechanics 722, 5-50.

Hara, T. \& Sullivan, P. P. 2015 Wave boundary layer turbulence over surface waves in a strongly forced condition. Journal of Physical Oceanography 45 (3), 868-883.

Hsu, C. T., Hsu, E. Y. \& Street, R. L. 1981 On the structure of turbulent flow over a progressive water wave: theory and experiment in a transformed, wave-following coordinate system. Journal of Fluid Mechanics 105, 87-117.

JANSSEN, P. 2004 The interaction of ocean waves and wind. Cambridge University Press.

Jefrereys, H. 1925 On the formation of waves by wind ii. Proc. Roy. Soc. 110A, 341-347

Jurman, L. A., Deutsch, S. E. \& McCready, M. J. 1992 Interfacial mode interactions in horizontal gasliquid flows. Journal of Fluid Mechanics 238, 187-219.

Kaffel, A. \& RiAZ, A. 2015 Eigenspectra and mode coalescence of temporal instability in two-phase channel flow. Physics of Fluids 27 (4), 042101 .

KolaAs, J. 2016 Getting started with HydrolabPIV v1.0. Preprint series. Research Report in Mechanics.

Kordyban, E. S. \& Ranov, T. 1970 Mechanism of slug formation in horizontal two-phase flow. Journal of Basic Engineering 92 (4), 857864

Lighthill, M. J. 1962 Physical interpretation of the mathematical theory of wave generation by wind. Journal of Fluid Mechanics 14 (3), 385-398.

Lin, M., Moeng, C., Tsai, W., Sullivan, P. P. \& Belcher, S. E. 2008 Direct numerical simulation of wind-wave generation processes. Journal of Fluid Mechanics 616, 1-30.

MAKIN, V. K. \& KudRYAVTSEV, V. N. 2002 Impact of dominant waves on sea drag. Boundary-Layer Meteorology 103 (1), 83-99.

Onorato, M., Residori, S., Bortolozzo, U., Montina, A. \& Arecchi, F.T. 2013 Rogue waves and their generating mechanisms in different physical contexts. Physics Reports 528 (2), 47 - 89, rogue waves and their generating mechanisms in different physical contexts.

Pan, Y \& Yue, D. K. P. 2014 Direct numerical investigation of turbulence of capillary waves. Physical review letters 113 (9), 094501.

Reul, N., Branger, H. \& Giovanangeli, J.-P. 2008 Air flow structure over short-gravity breaking water waves. Boundary-layer meteorology 126 (3), 477-505.

SAnchis, A., Johnson, G.W. \& Jensen, A. 2011 The formation of hydrodynamic slugs by the interaction of waves in gas-liquid two-phase pipe. I. J. of Multiphase flow 37, 358-368.

Siddiqui, M. H. K. \& Loewen, M. R. 2006 Detecting microscale breaking waves. Measurement Science and Technology 17 (4), 771.

Siddiqui, M. H. K. \& Loewen, M. R. 2010 Phase-averaged flow properties beneath microscale breaking waves. Boundary-Layer Meteorology 134 (3), 499-523.

Sullivan, P. P., Banner, M. L., Morison, R. P. \& Peirson, W. L. 2018 Turbulent flow over steep steady and unsteady waves under strong wind forcing. Journal of Physical Oceanography 48 (1), 3-27.

Toffoli, A, Proment, D, Salman, H, Monbaliu, J, Frascoli, F, Dafilis, M, Stramignoni, E, Forza, R, Manfrin, M \& Onorato, Miguel 2017 Wind generated rogue waves in an annular wave flume. Physical review letters 118 (14), 144503.

Tzotzi, C. \& AndRItsos, N. 2013 Interfacial shear stress in wavy stratified gas-liquid flow in horizontal pipes ". I. J. Multiphase Flow 54, $43-54$

Veron, F., Saxena, G. \& Misra, S. K. 2007 Measurements of the viscous tangential stress in the airflow above wind waves. Geophysical Research Letters 34 (19).

Woods, B. D., Hurlburt, E. T. \& Hanratty, T. J. 2000 Mechanism of slug formation in downwardly inclined pipes. International journal of multiphase flow 26 (6), 977-998.

Wu, Y. \& Christensen, K. T. 2006 Population trends of spanwise vortices in wall turbulence. Journal of Fluid Mechanics 568, 55-76.

YANG, D. \& SHEN, L. 2010 Direct-simulation-based study of turbulent flow over various waving boundaries. Journal of Fluid Mechanics 650, 131-180. 
Zonta, F, Onorato, M \& Soldati, A 2016 Decay of gravity-capillary waves in air/water sheared turbulence. International Journal of Heat and Fluid Flow 61, 137-144.

Zonta, F, Soldati, A \& Onorato, M 2015 Growth and spectra of gravity-capillary waves in countercurrent air/water turbulent flow. Journal of Fluid Mechanics 777, 245-259. 